\title{
Concepción de conocimiento: ¿Obstáculo/facilitador en la formación universitaria de líderes comunitarios?
}

\author{
Rodrigo Araya Campos \\ Pontificia Universidad Católica de Valparaíso. Escuela de Periodismo. Chile.
} Email: raraya@ucv.cl

\section{Cristina Julio Maturana}

Pontificia Universidad Católica de Valparaíso. Escuela de Pedagogía. Chile. Email: cristina.julio@ucv.cl

\section{Patricia Moggia Münchmeyer}

Pontificia Universidad Católica de Valparaíso. Escuela de Pedagogía. Chile. Email: patricia.moggia@ucv.cl

\section{María Soledad Vargas Carrillo}

Pontificia Universidad Católica de Valparaíso. Escuela de Periodismo. Chile. Email: maria.vargas@ucv.cl

Resumen: ${ }^{1}$ El artículo presenta algunos resultados de la Investigación titulada: "Formación de líderes comunitarios en la Pontificia Universidad Católica de Valparaíso (PUCV): hacia una concepción más pertinente” desarrollada durante 2012 por un grupo de académicos y académicas del “Diplomado en Educación y Cultura” que impartió la PUCV en 2011 a Directoras de Educación de los campamentos que apoya ‘Techo en la Región de Valparaíso’. De la experiencia surge como problema la pertinencia de la concepción de conocimiento de la propuesta formativa. Es así como el estudio, de carácter cualitativo interpretativo, asume como objeto de estudio la concepción de conocimiento y su pertinencia en la formación de estas líderes, y su unidad de análisis es el propio diplomado. Sustentados en la discusión empírica emanada de las voces de los actores y de documentos institucionales, y de la discusión teórica emergente de las mismas, se da cuenta de los imaginarios sobre el conocimiento.

Palabras clave: conocimiento, formación universitaria de líderes comunitarios, conocimiento situado. 


\title{
Concept on knowledge: Obstacle/facilitator in university education of community leaders?
}

\begin{abstract}
This paper presents some of the results of the research "Education of Community Leaders in Pontificia Universidad Católica de Valparaíso (PUCV): towards a more appropriate conception", realized during 2012 by a group of academics of the "Education and Culture Certification Program", offered by PUCV in the year 2011 to Education Directors of the camps supported by 'Roof in the Valparaiso Region'. From this experience, arises the problem of the appropriateness of the conception of knowledge in the educational proposal. Thus, this qualitative, interpretative research focuses on the knowledge conception and its appropriateness in the education of these leaders, and its unit of analysis is the Program itself. Based on the empirical discussion that arises from the voices of the actors, on the institutional documents and the theorical discussion that comes from them, the imaginaries about knowledge are reported.
\end{abstract} knowledge.

Key words: knowledge, university education of community leaders, situated

\section{Concepção de conhecimento: Obstáculo/facilitador na formação universitária de líderes comunitários?}

Resumo: O artigo apresenta alguns resultados da pesquisa intitulada "Formação de líderes comunitários na Pontifícia Universidade Católica de Valparaíso (PUCV): para um conceito mais relevante”, desenvolvido em 2012 por um grupo de acadêmicos do “Diplomado em Educação e Cultura”, que deu a PUCV em 2011 aos Diretores da Educação nas favelas que apoia o Programa "Um Teto para Chile" na Região de Valparaíso. Desde esta experiência surge como problema a pertinência do conceito do conhecimento da proposta de formação. Assim, o estudo de caráter qualitativo e interpretativo, assume como objeto de pesquisa a concepção do conhecimento e sua relevância para a formação desses líderes e sua unidade de análise é o próprio diplomado. Baseado no debate empírico que emana das vozes dos atores, de documentos institucionais e da discussão teórica emergente das mesmas, dá-se conta dos imaginários sobre o conhecimento.

Palavras-chave: conhecimento, formação universitária de líderes comunitários, conhecimento situado.

$* * *$

\section{Universidad, conocimiento y formación de líderes comunitarios: ¿un problema?}

El presente artículo da cuenta de los resultados de una investigación sobre la pertinencia de la concepción de conocimiento que sustenta la formación de líderes comunitarios para la resolución de problemas complejos en educación y cultura en contextos de pobreza.

Este cuestionamiento surge tras la dictación de un diplomado que un grupo de profesores de la PUCV (entre los cuales se cuentan los cuatro investigadores), impartió a directoras de educación de campamentos, apo- 
yados por Techo², en el Gran Valparaíso. Ello, por cuanto fue visto por los investigadores como una tensión por el encuentro entre dos tipos de conocimiento: el de la academia, y el de líderes comunitarios, mediados por una tercera, la de Techo.

Por lo tanto, aparece relevante conocer si la concepción de conocimiento actúa como obstáculo/facilitador, en dicha formación y de este modo, poder avanzar hacia la construcción de un modelo formativo más pertinente.

Al mismo tiempo, esta investigación se produce en momentos en que la función social de la universidad recibe diversos cuestionamientos, toda vez que parece estar más centrada en la formación de personas (especialmente, de profesionales), que en el modelo de conocimiento que esta genera y difunde.

Si tomamos como ejemplo el actual cuestionamiento que sufre el sistema de educación superior en Chile, éste se gatilla por la inequidad que hay en el acceso a la formación universitaria. Por lo tanto, las demandas de cambio quedarían resueltas si se dispusiera de mecanismos de ingreso que no resulten discriminatorios para los postulantes que provienen de los sectores sociales en situación de pobreza.

Al cuestionar la formación, y no la epistemología que difunde, se corre el riesgo de dejar el modelo de conocimiento que difunde, fuera de la crítica al modelo universitario. Algo de esto ocurre en el caso chileno: en bibliografía reciente que examina el caso de las universidades (González, 2011; Esquivel, 2007; Donoso y Schiefelbein, 2007; Rodríguez-Ponce, 2011), no se encuentran preguntas por el lugar que tiene el conocimiento en la crisis que enfrenta la educación superior.

Ello, a pesar que la noción de que el conocimiento es valóricamente neutro, ha recibido cuestionamientos tanto epistémicos (Dilthey, 1978, Gadamer, 1993), como del quehacer de las ciencias (Feyerabend, 1989, Lakatos, 2001, Wallerstein, 2007), lo que incluso ha llevado a cuestionar la universalidad que el conocimiento humano puede alcanzar (Chakrabarty, 1992).

\section{¿Desde dónde discutir el problema? Algunos referentes teóricos}

El marco referencial desde el cual se sustenta el estudio ha sido organizado en torno a cuatro ejes: el conocimiento situado; su relación con la formación de líderes comunitarios, la posibilidad de re-definir el rol social de la universidad y los desafíos para asumir una formación más pertinente. 


\section{Conocimiento situado: ¿ ¿una alternativa epistémica?}

Los cuestionamientos planteados han permitido proponer que el conocimiento tiene condición de situado, ya que se entiende que la producción de conocimiento no sólo se realiza dentro de un horizonte temporal, sino también de uno espacial. Es decir, no sólo en un ahora, también en un aquí.

El conocimiento situado ofrece un ángulo de mirada para replantearse el rol social de la universidad, en tanto esta institución posee dispositivos de generación y transmisión de conocimiento que tampoco son valóricamente neutros. Dicho de otro modo, su labor puede ser sometida a crítica desde la perspectiva del conocimiento situado (Walsh, 2007), y desde allí analizar, bajo una perspectiva crítica, la pertinencia de su labor, en el entendido que el conocimiento, como otras obras humanas, ocupa una posición geopolítica (Mignolo, 2001).

A diferencia de lo que ocurre en Chile, en el resto del continente se observa una significativa creación de universidades interculturales e indígenas (Mato, 2009), cuyo interés epistémico es recuperar o generar racionalidades que se alejen de la hegemónica modernidad eurocéntrica (Mignolo, 2001), para avanzar hacia una diversidad epistémica.

Para lograr este objetivo, se requiere un cambio en las unidades del conocimiento: salir de una orientación a la construcción de conceptos, para centrarse en la construcción de imaginarios (Kozlarek, 2007). Esto es, generar un diálogo entre los conceptos disponibles, y la práctica social bajo la que se pensaron los conceptos. Ello, en el entendido que todo conocimiento es resultado de una actividad práctica que se da al interior de una determinada concepción de naturaleza, concepción de la que emanarán los modos adecuados de vincularse con ella (Escobar, 1993). Es decir, al abandonar la oposición radical entre naturaleza y cultura, se comprende el conocimiento como situado, pues si la naturaleza es vista como una construcción cultural (Escobar, 1993) deja de percibirse la existencia de un único modelo de conocimiento, sino más bien la imposición de esta idea desde los nodos hegemónicos de producción de conocimiento.

\section{Formación de personas y conocimiento situado}

La importancia de formar personas bajo otros imaginarios ${ }^{3}$ radica en que de este modo, se puede contar con profesionales capaces de trabajar por la construcción de un orden social otro, es decir, diverso al predominante. Así, por ejemplo, el surgimiento de las universidades indígenas e interculturales en América Latina (Mato 2009 y 2010) se explica, entre otros factores, como respuesta a la necesidad de contar con profesionales que puedan cumplir dos labores. Primero, favorecer el diálogo con las instituciones, especialmente las del Estado, dado que ellas no están preparadas 
para el encuentro intercultural. Y en segundo término, contar con profesionales que pudieran trabajar por la construcción del orden social propio de esa identidad.

Dicho de otro modo, la universidad como institución si se pone al servicio de un conocimiento que no reconoce lugar (o imaginarios), prepara profesionales para un orden social que tampoco reconoce lugar. Para justificar este gesto, se requiere contar con un imaginario que ubique a este conocimiento como superior. Esto nos ubica en la conocida crítica al eurocentrismo (Lander, 1993; Mignolo, 2001), es decir, a un conocimiento que al presentarse a sí mismo como trascendente a cualquier lugar, se asume como resultado del proceso evolutivo de la especie humana (por lo tanto, se le naturaliza), y en consecuencia, se trataría del conocimiento de mayor refinamiento que existe en el planeta.

Asimismo, si tomamos prestada la metáfora de la distinción de Bourdieu (1989), se puede sostener que la universidad actúa como un dispositivo de distinción de la racionalidad predominante. Esto se alcanzaría gracias a dos acciones complementarias: la naturalización o deshistorización del conocimiento que generan y difunden, y la legitimación social que tiene su rol de formación de profesionales, es decir, de personas que alcanzan distinción gracias al conocimiento que adquieren en la universidad. Así, la universidad, como institución, lograría difundir un conocimiento legitimado, y por esa vía, otorgaría legitimidad a las personas (profesionales) que forma.

\section{¿Es posible re-definir el rol social de la universidad? Su vinculación con líderes comunitarios}

Lo planteado en el acápite anterior permite afirmar que la universidad está en posición de aportar a dos dimensiones de la justicia social: de redistribución (por lo tanto, de orden material) y de reconocimiento (Fraser, 2000). A la de redistribución, en tanto cuenta con condiciones materiales para que todas las personas con interés y capacidad puedan ingresar, permanecer y egresar de ellas.

Y de reconocimiento, pues la universidad, mediante su certificación, otorga re-conocimiento social que autoriza para desempeñar con legitimidad una determinada profesión. En este proceso, no sólo legitima a la persona poseedora del certificado, sino también los conocimientos, en tanto imaginarios, que sustentan esa práctica profesional.

Por lo tanto, la universidad puede aportar a la justicia social mediante la ampliación del registro de los conocimientos (en tanto imaginarios) a los que otorga legitimidad ${ }^{4}$. De lo contario, favorece la preservación, fortalecimiento y ampliación de un modelo único de desarrollo, quedando ubicada como una verdadera universidad fordista que contribuye a la 
homogeneización de lenguajes para posibilitar el mercado sin fronteras (Manzano- Arrondo, 2012).

Para avanzar en esta línea, parece clave la relación universidad-comunidad. Esto, que en las universidades chilenas se conoce con el nombre de vinculación con el medio, es lo que permitiría articular necesidades sociales de la población (las que son resultado de una construcción) con el conocimiento generado por la universidad: "los tiempos que corren requieren de una universidad comprometida con la vida comunitaria, con las necesidades de los ciudadanos que habitan la comunidad, con el ejercicio de un rol de democratización del saber basado en el principio que reconoce que el conocimiento se construye socialmente y en base al respeto a las diversidades de cada grupo humano” (Tonon 2012: 517).

\section{Universidad: El desafío de una formación pertinente de líderes comunitarios}

Considerando la posibilidad de nuevas formas de vincularse con líderes comunitarios, uno de los desafíos mayores que enfrenta el conocimiento que la universidad posee, es la formación de ellos y ellas. Esto, en el entendido que se trata de personas que, con frecuencia, no han completado su escolaridad, por lo que se puede afirmar que no han sido formadas en la racionalidad predominante. Por lo tanto, se trataría, a ojos de la educación formal, de personas que no cuentan con los conocimientos previos que la universidad valora. De modo tal, que los procesos de enseñanza de la universidad no están pensados para ellos, y los contenidos presentados no resultan necesariamente apropiados para los contextos socioculturales en que los líderes los van a desarrollar.

Para evitar que la situación descrita se transforme en un obstaculizador del proceso formativo referido, a juicio de los investigadores parece recomendable no obviar la pregunta por la pertinencia. Esta es la que permitiría distinguir si esta formación disciplina ${ }^{5}$ en un conocimiento no pertinente a líderes comunitarios, o, por el contrario, contribuye a que desde su modo de conocer se puedan construir nuevas estrategias analíticas y metodológicas para otorgar pertinencia a su labor.

Se trata por lo tanto de una prevención epistémica: si el mejor conocimiento es aquel que otorga respuestas más apropiadas a un problema (es decir, a carencias que se perciben en un lugar determinado bajo un imaginario determinado), aparece de mejor calidad una epistemología que logra integrar visiones diversas respecto de aquella que excluye otras visiones para imponer la propia (Morin, 2001).

Esto significa dar relevancia al conocimiento como una cuestión que surge desde el lugar (Escobar, 1993). Esto, por cuanto la comprensión antropológica del lugar lo entiende como el resultado de la relación dinámi- 
ca de una sociedad dada con la naturaleza, para alcanzar un determinado concepto de bienestar (Ysern, 2007). Esto permite sostener que el conocimiento pertinente, o situado, es aquel que se pone al servicio de una sociedad determinada y su noción de futuro.

\section{Metodología}

Sustentada en una epistemología de pensamiento complejo (Morin, 2001 y Morin y otros, 2003) la concepción de conocimiento como objeto de estudio, es asumida desde su multidimensionalidad, articulando dominios disciplinarios diferentes. En coherencia y para develar dichas concepciones se optó por un diseño de carácter cualitativo con dos métodos complementarios (documental y entrevistas) y de desarrollo paralelo, asumiendo como unidad de análisis el Diplomado de Educación y Cultura.

La opción documental se sustenta en que los textos institucionales escritos pueden ser considerados una modalidad de producción material cultural (Hodder, 2000) en la medida en que proponen cosmovisiones y regulan ciertas formas de acción en cada institución. El corpus documental se constituyó de 28 documentos (11 de Techo y 17 de la PUCV) respondiendo a dos criterios, uno diacrónico y otro sincrónico referido a la oficialidad del contenido. En el caso de Techo, se asumió la etapa fundacional y la actual considerando memorias, libros, manuales e informes del período 20062012. En el caso de la PUCV se abarcó la etapa fundacional, 1928; el de la reforma, 1966-1971 y el contemporáneo, 2008-2012. En el corpus de esta institución se consideró el discurso Inaugural de cada año Académico y la cuenta pública que el Rector rinde ante el pleno de la Universidad.

En este punto, es necesario hacer la prevención, que asumimos que el discurso de la universidad no es único ni uniforme, no obstante, hemos recogido como fuente documental aquellos discursos que la propia institución ha ido legitimando a través del tiempo como su propio discurso, como una forma de ir dejando huella, registro a través de los dichos de sus autoridades y académicos reconocidos por su sapiencia o prestigio social: rectores, cancilleres universitarios, profesores eméritos entre otros.

Las entrevistas siguieron el criterio de informantes claves, procurando la participación de las distintas personas involucradas en la formación de líderes comunitarios: Líderes Comunitarios (Directoras de Educación y otros representantes de los campamentos), representantes de la PUCV (Profesores del Diplomado que no participan de la investigación y otros académicos) y representantes de Techo (Directivos regionales y voluntarios). Tal como se presenta en la Tabla I, los extractos discursivos emanaron de 10 entrevistas individuales en profundidad del tipo activo-reflexivas (Sisto \& Fardella, 2008) realizadas a 4 líderes, 3 profesores y 3 directivos de Techo. Estas fueron complementadas, con posterioridad, con 3 entrevistas grupales de carácter interinstitucional en las que participaron 4 líderes, 6 representantes de la PUCV y 7 de Techo. 
Tabla I: Participantes

\begin{tabular}{|c|c|c|c|c|c|}
\hline & Informantes Claves & $\begin{array}{c}\text { Entrevistas } \\
\text { individuales } \\
\text { (10) }\end{array}$ & $\begin{array}{c}\text { Entrevista } \\
\text { grupal 1 }\end{array}$ & $\begin{array}{c}\text { Entrevista } \\
\text { grupal } 2\end{array}$ & $\begin{array}{c}\text { Entrevista } \\
\text { grupal } 3\end{array}$ \\
\hline$\stackrel{9}{\frac{\pi}{6}}$ & $\begin{array}{l}\text { Directivo Techo(DT) } \\
\text { Voluntario Techo(VT) }\end{array}$ & $\begin{array}{l}\text { (DT-1) } \\
\text { (DT-2) } \\
\text { (DT-3) }\end{array}$ & $\begin{array}{l}\text { (VT-1) } \\
\text { (DT-4) } \\
\text { (DT-5) }\end{array}$ & $\begin{array}{l}\text { (VT-2) } \\
\text { (DT-6) }\end{array}$ & $\begin{array}{l}\text { (VT-3) } \\
\text { (DT-1) }\end{array}$ \\
\hline 己 & $\begin{array}{l}\text { Académica Universidad } \\
\text { Profesora Diplomado (AU- } \\
\text { PD) } \\
\text { Directivo Universidad(DU) } \\
\text { Académico Universidad } \\
\text { (AU) }\end{array}$ & $\begin{array}{l}\text { (AU-PD-1) } \\
\text { (AU-PD-2) } \\
\text { (AU-PD-3) }\end{array}$ & $\begin{array}{l}(\mathrm{DU}-1) \\
(\mathrm{AU}-1)\end{array}$ & $\begin{array}{l}(\mathrm{DU}-2) \\
(\mathrm{AU}-2)\end{array}$ & $\begin{array}{l}\text { (AU-PD-3) } \\
\text { (AU-3) }\end{array}$ \\
\hline 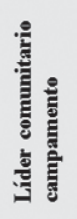 & $\begin{array}{l}\text { Líder Comunitaria Directora } \\
\text { Educación Campamento con } \\
\text { Diplomado (LCDEC-D) } \\
\text { Líder Comunitario } \\
\text { Campamento (LCC) } \\
\text { Líder Comunitaria Directora } \\
\text { Educación Campamento } \\
\text { (LCDEC) }\end{array}$ & $\begin{array}{l}\text { (LCDEC-D-1) } \\
\text { (LCDEC-D-2) } \\
\text { (LCDEC-D-3) } \\
\text { (LCDEC-D-4) }\end{array}$ & (LCDEC-D-3) & $\begin{array}{c}\text { (LCDEC-D- } \\
4) \\
(\text { LCC-1) }\end{array}$ & (LCDEC-1) \\
\hline
\end{tabular}

Las 13 entrevistas cualitativas facilitaron aproximarse a la perspectiva del otro posibilitando una interpretación más cercana a los planteamientos de los participantes (Mella, 1998) y una construcción conjunta de significados (Hernández, 2003), más aún cuando los participantes muestran diversos imaginarios al interior de cada una de las instituciones y entre líderes comunitarios.

El análisis interpretativo y obtención de resultados se realizó a través de un proceso de codificación y categorización conceptual en torno a cuatro preguntas: ¿qué entiende por conocimiento?, ¿qué tipo de conocimientos reconoce?, ¿qué papel social le atribuye al conocimiento?, ¿qué aporte le atribuye al conocimiento para la superación de las injusticias sociales? Ellas permitieron desarrollar la síntesis del objeto de estudio a través de tres sistemas de codificación articulados entre sí que incluyeron codificación abierta, codificación axial y codificación selectiva (Goetz \& Le Compte, 1988; Osses, et al 2006) y del proceso de triangulación respectivo. Además, para la codificación de las entrevistas se empleó el software Atlas Ti.

Así, la teoría fue emergiendo desde los datos, manteniendo el referente empírico (Mella, 1998 y Mayan, 2001) y permitiendo el desarrollo de categorías significativas asociadas a la búsqueda de respuestas a las preguntas orientadoras y sustentándose en el carácter reflexivo y permanente de la investigación social (Mella 1998) que mantuvimos los autores. 


\section{Resultados: en búsqueda de una noción pertinente de conocimiento}

Tal como se indicó en la metodología, los resultados dan cuenta de los imaginarios que emergieron en torno a las cuatro preguntas sobre conocimiento.

\section{¿Qué entiende por conocimiento?}

Las respuestas emergentes a esta pregunta emanaron desde dos ámbitos: desde la Ontología y desde la Ética.

A partir de la perspectiva Ontológica, para la Universidad el conocimiento es visto como una construcción social: "El conocimiento en su sentido amplio, la representación del mundo y de su propia realidad, han permitido al hombre avanzar hacia el dominio de la naturaleza y, desde ahí, simultáneamente, preguntarse acerca de su propio origen y destino, individual y colectivamente" (Zabala 1966: 5). Esta construcción social está mediada por una relación dialógica, la cual lleva a una concepción amplia de educación y a una concepción amplia de aprendizaje: "la educación no es especialmente la educación en el colegio, que nosotros nos estamos educando todos los días en la vida cotidiana, desde que nacimos, algunos creen que la educación viene del colegio nomás, y nosotros en la casa estamos educando a nuestros hijos, desde el momento del nacimiento hasta que se fallece. Todos los días nos educamos y nosotros siempre nos estamos educando, ellos aprenden de nosotros y nosotros de ellos, esa es una educación también, eso nos enseñaron eso no lo tenía claro” (LCDECD-3).

En este mismo ámbito ontológico, el conocimiento se asume como algo natural (dado), por lo tanto, se percibe como neutro. Esto es visto por la Universidad y por el Techo: “Tiene que ver cuando uno sabe más es más persona es más aporte en todo sentido, porque uno tiene más confianza, porque uno tiene nuevos conocimientos que antes...” (DT-3). Asimismo, se asume que es superior a la experiencia, esto último, desde la percepción de la universidad: "La Universidad, lugar en que la cultura del hombre alcanza un grado superior de conciencia reflexiva, debe preguntarse continua y valientemente, acerca de su ser y por ende acerca de su función. Ante nuevos fenómenos que van cambiando la faz del mundo, la Universidad debe formularse muchas preguntas, de orden teórico y práctico, a las cuales no es siempre fácil contestar" (Zabala 1966: 4).

En consecuencia, este conocimiento ordena la realidad, en cuanto es el que permite poner nombre a las cosas: "Era esta tensión entre que la clase avanzaba y te dedicabas a reflexionar o a ver cómo algunos elementos teóricos que ellas también estaban expectantes de aquello. De poner el nombre teórico de lo que ya sabían, por ejemplo, yo partía como desde las experiencias previas y claramente ellas tenían conocimiento, pero yo tam- 
bién creía que ellas querían ponerle el nombre a eso que sabían. El teórico. Y para eso se requiere un tiempo de poder (...) como hacer la unión entre su experiencia, sus saberes y la teoría” (AU-PD-2).

Por último, en esta misma visión, se asume por los tres actores: Universidad, Techo y Líderes comunitarios que el conocimiento habilita: "la habilitación social es el signo más claro del desarrollo de un país e involucra en este proceso áreas tan importantes como lo son la justicia, la salud, el trabajo, el emprendimiento y la educación, siendo esta última, de acuerdo a nuestros gobiernos y a los organismos internacionales, una de las áreas en que nuestra población se encuentra más vulnerable, así como ha demostrado ser el motor y piedra angular del desarrollo de una sociedad en cuanto aplaca los niveles agudos de desigualdad social, cultural y económica que existe en un país" (Techo 2011-2012: 1). De este modo, transforma a las personas en interlocutores válidos para dialogar con el orden establecido: "con la Universidad nosotros podemos saber buscar apoyo, cómo podemos hablar con las otras personas, eso no lo sabía, y ahora ya podemos, ya sabemos cómo comunicarnos con la gente, tener una mayor comunicación con los vecinos que no llegue a discusión. Hablar ante la gente, saber buscar las necesidades que tiene cada uno de ellos y desarrollarlas y fortalecerlas. Antes nosotros buscábamos no más la directiva una reunión y listo. Ahora como directora busco las necesidades de cada uno de ellos, porque si necesitan algo ahí estoy, y gracias a la Universidad que me ayudó a esto, a ser mejor director y he podido buscar apoyo" (LCDEC-D-3).

Desde la perspectiva Ética, para la Universidad el conocimiento se complementa con otros valores humanos: "Si se cultiva la inteligencia con el conocimiento de la verdad científica, hay que cultivar también la voluntad y el corazón con las austeridades de la virtud" (Castro 1928: 1). Por lo mismo, humaniza en la medida que favorece la conformación de una persona autónoma y que goza de libertad: "el conocimiento propiamente educativo es aquel que modifica la forma de vida del educando, situándolo en una nueva dimensión de su relación con el entorno y principalmente consigo mismo. (...) educada es la persona que se transformó interiormente en una persona que se posee mayor y más profundamente a sí mismo y, así puede actuar en su entorno de un modo más libre y señorial" (Lorenz, 2010: 5).

En este mismo ámbito ético, el conocimiento se asume como poder, pues desde la Universidad sirve para dominar la naturaleza. Asimismo, es visto por los Líderes comunitarios como útil para obtener validación en la comunidad: "ellos (los hijos) van a ir a la universidad, pero ella ahora que está saliendo de cuarto medio igual como que se viene difícil, por los pagos. Y la carrera de ella igual es cara y yo la veo muy, muy, muy entusiasmada en eso, entonces no quiero matarle la ilusión, por eso le digo: postula a todas las becas habidas y por haber" (LCDEC-D-4). En consecuencia, para este grupo sirve para obtener distinción social.

Finalmente, desde una lógica de poder, para los mismos Líderes comunitarios marca la oposición público-privado, invisibilizando el conoci- 
miento experiencial: “en realidad no nos enseñaban a ser líderes, sino que conversábamos el liderazgo entre todas, todas las experiencias nos servían, entonces tomábamos las experiencias de cada una y después nos dimos cuenta que a la final igual nosotras sabíamos mucho, sabíamos mucho de liderazgo y no lo teníamos asumido.”(LCDEC-D-4).

\section{¿Qué tipos de conocimientos reconoce?}

Tras el análisis de los discursos de nuestras fuentes, podemos observar que emergen tres grandes tipos de conocimiento, que se establecen como dicotomías: teórico v/s práctico, cotidiano v/s científico y por último, situado v/s universal.

En un primer nivel, el conocimiento es asumido desde la Universidad como teórico, por lo cual se le otorga una jerarquía superior, pues se aspira a ser y a hacer ciencia: "Si se cultiva la inteligencia con el conocimiento de la verdad científica, hay que cultivar también la voluntad y el corazón con las austeridades de la virtud” (Castro, 1928: 1).

El conocimiento científico en la Universidad para algunos humaniza, pues permite alcanzar la verdad y el sentido del ser en el mundo: "Este conocimiento científico objetivo de la existencia concreta, es para nosotros de primera importancia, puesto que nos iluminará en toda nuestra actividad universitaria. Daremos, en consecuencia, prioridad al desarrollo de las ciencias sociales básicas como instrumento insustituibles para el conocimiento de la realidad concreta” (Zabala, 1966: 5).

Para algunos profesores de la Universidad, se considera que este conocimiento, a veces, deshumaniza: "Sería muy duro reconocer la pérdida de valores humanos tan importantes en aquellos que han recibido una formación privilegiada y están llamados a ser líderes” (Ibid: 6). Algunos profesores perciben que al trabajar en la Universidad, en muchas ocasiones, se pierde el contacto con la realidad: “como educadora siempre trabajé en las poblaciones, y el día en que decidí estar en la Universidad, decidí no perder de vista el trabajo en terreno para no olvidarme del por qué soy educadora, porque de lo contrario uno se olvida. La universidad es mucha investigación, bastante abstracta, luego uno empieza a hablar de lo que fue como educadora para transmitir esa experiencia mientras hace clase (...) Y la única manera de vivir esa transformación es ser parte de esto aún y no ser alguien que está hablando (...) de cosas que fueron” (AU-PD-2).

Algo similar es percibido por el Techo. Esta condición deshumanizadora del conocimiento se produciría por la excesiva división disciplinar. Lo que, a su vez, desde la perspectiva de Techo generaría un conocimiento encapsulado: "Yo estudio Historia y el ambiente de intelectualidad que algunos profesores o personas que llevan 30 años trabajando en eso, tienden a crear muchas veces a que en las mismas universidades se forme como un núcleo cerrado en torno a lo que se sabe o los conocimientos. Cuesta mucho que la gente externa llegue a una universi- 
dad a aprender" (VT-3). Finalmente, en esta acción deshumanizante el conocimiento es percibido como jerárquico. Lo anterior, trae como consecuencia el enfocarse exclusivamente en el individuo.

Para Techo el conocimiento es visto como práctico y por ende, es considerado superior para este actor: "habían conceptos muy teóricos como la definición del concepto de aprendizaje que es muy legítimo (...) y cómo deben interpretar ellas el acto educativo o la transmisión de conocimientos, pero por otro lado cuando un dirigente social está dedicando tiempo que podría estar dedicando a generar algún ingreso para su familia , si tuviera un valor agregado como puede ser alguna actitud o nueva herramienta para que pueda aplicar en su vida diaria , al final la inversión que ya está haciendo en tiempo se puede traducir también en una ganancia más concreta” (DT-3).

Desde esta perspectiva, se considera que el conocimiento es habilitador: "No basta con llevar a los niños a la escuela, eso no garantiza una educación que habilite a la persona para alcanzar sus objetivos económicos y sociales, y para adquirir las competencias, los conocimientos, los valores y las aptitudes que hacen surgir una ciudadanía responsable y activa” (Techo, 2011: 50). Afirmación que también es compartida por algunos profesores de la Universidad.

Asimismo, el conocimiento práctico permitiría vincular con la realidad: "el aporte del Techo puede ser muy grande, te permite tener un acercamiento a la realidad o a la actividad misma del dirigente, que eventualmente podría traducirse en mayor utilidad al mismo diplomado; si tú complementas la vida cotidiana de la participante al diplomado, que por un lado te la da a través de la experiencia, por otro lado, te la da el Techo a raíz de miles de experiencias que vive cada uno de los voluntarios que van semana a semana a los campamentos, creo que puede ser mucho más útil si se complementan esos tres pilares, por un lado lo académico que aporta la universidad, la experiencia personal que puede aportar el mismo beneficiado del programa y un Techo para Chile" (DT-3).

Finalmente, desde Techo y desde algunas voces de la Universidad se lo reconoce como emprendimiento. En efecto, para algunos directivos de la Universidad, esta visión del conocimiento sería fundamental y, a la vez, un medio para salir de la pobreza: "Nos hemos empecinado en trabajar con la gente del Techo, en poder descubrir, impulsar el emprendimiento en las personas, sean o no sean líderes, por supuesto que tiene que ver con las capacidades y habilidades emprendedoras (...) Y en eso nosotros aportamos como Universidad, herramientas y ayuda o apoyo que puede ser, y es lo que vamos a hacer hacia adelante también, de acompañar y fortalecer todas las capacidades que puedan tener emprendedoras tanto líderes como no líderes en el campamento y creemos que ahí está como un nicho que a lo mejor no se ha abordado nunca y que tiene ver cómo las personas que están en los campamentos puedan descubrir esto, puedan reinventarse, puedan generar empleos, puedan aumentar su ingreso mensual, pueden ver 
otra realidad que es que trasciende más allá de los límites físicos de un campamento" (DU-1).

En cuanto habilitador, para la Universidad y para los Líderes comunitarios se lo comprende como formador. Asimismo, en este nivel para los tres actores se asume el conocimiento como capacitación.

Es importante señalar, que dentro de esta primera tipología se produce una transición entre el conocimiento teórico y práctico, dando origen a uno de tipo teórico-práctico, esto es visto esencialmente por el Techo: “el desafío creo que está justamente en ese espectro académico como de alto nivel, bajarlo entre comillas, no me gusta ese término, pero es como aterrizarlo, no es bajar el nivel, sino incorporarle el sentido práctico y aplicado que tiene que tener en la realidad de los líderes comunitarios” (DT-4). Algo similar ocurre entre la formación y la capacitación, pues tanto para el Techo como para los Líderes comunitarios, el conocimiento se percibe como formación-capacitación.

En un segundo nivel de tipología, se comprende al conocimiento como cotidiano v/s científico. Para los líderes comunitarios se valora el conocimiento práctico, el cual es experiencial, surge tras la experiencia de vida. Por lo mismo, cómo es una experiencia que se da en el hogar, proviene del espacio íntimo, por lo tanto, es un conocimiento que proviene desde lo privado. Es necesario, hacer hincapié que este conocimiento cotidiano es considerado inválido tanto para la Universidad como para el Techo; pues tiene una valoración desde la Ética, como algo inferior o ilegítimo. Por lo mismo, por ser considerado inválido se lo asume con una dimensión política, pues no permitiría dialogar con las autoridades o interactuar con la élite o grupos dominantes.

En este mismo nivel, el conocimiento científico para la Universidad es visto como el conocimiento válido: "Buscamos ansiosamente la Verdad y tenemos la certeza que a medida que progresamos en su búsqueda, vamos perfeccionando nuestro conocimiento” (Zabala, 1966: 1). En consecuencia, es en este espacio donde estaría radicado y se lo reconoce desde el ámbito público.

Finalmente, en un tercer nivel, encontramos que el conocimiento se percibe como universal: "Y esa mirada más local es compleja porque la universidad apunta, justamente, a la universalidad, a lograr establecer, ojalá, un modelo, pero no necesariamente ese modelo va a ser lo que se va a necesitar en cada campamento” (DT-4). En consecuencia, no hay expresiones que apunten hacia lo que acá se ha definido como conocimiento situado.

\section{¿Qué papel social le atribuye al conocimiento?}

A partir de los discursos de las fuentes analizadas, surgen cuatro tipos de papeles: uno atribuido por los pobladores según etapas etáreas; el 
segundo, transformador de vidas; otro sociopolítico y el cuarto, un papel intelectual.

Las voces de los líderes comunitarios atribuyen papeles distintos al conocimiento según etapas etáreas. Para los jóvenes el conocimiento permitiría alcanzar movilidad social: "Yo lucho todos los días con decirle que estudien, que estudien (...) si tú estudiai vai a tener un buen sueldo, vai a tener una casa, vai a tener un auto, vai a tener tus cosas” (LCDEC-1); en cambio para los adultos, estaría al servicio del liderazgo y hacia la construcción de comunidad, para la superación de la condición de pobreza: "para saber hacer proyectos de, para gestiones de proyectos que eso nos sirve harto, porque uno para conseguir algo para la comunidad, algo material, o un paseo o ir a algún lado, tienes que gestionar un proyecto para hacer eso" (LCDEC-D-3).

El papel transformador de vidas del conocimiento atribuido por la Universidad y Techo, evidencia dos tipos de transformaciones una individual y otra comunitaria. En la individual, Techo plantea que el conocimiento y el acceso a la formación empodera, le da un sentido de apropiación y potencia las capacidades del individuo: “lo que más me llamó la atención primero fue el empoderamiento que le dio [a las líderes comunitarias] el haber hecho el diplomado en la Universidad” (DT-3). Esta visión es compartida por las propias Líderes, quienes manifiestan que hay un antes y un después en su proceso personal "no tenía ni la capacidad de hablar con personalidad como estoy hablando ahora” (LCDEC-D-3). Para Techo el empoderamiento es visto como una condición necesaria, una herramienta fundamental para aportar a la comunidad: "en la medida en que la directora entendió bien su rol, también fue capaz de mejorar su intervención con la comunidad" (DT-1).

El empoderamiento se aprecia en dos espacios: uno público y otro privado. Lo público, señalado por los tres actores, estaría entendido como lo que se requiere saber para tener una mejor interlocución con las autoridades; lo que se evidencia en algunas voces de la universidad indican "en una persona que va asumir un rol de coordinación de algo en una comunidad, hoy día eso [el aprendizaje de las TIC] es esencial, o si no ¿cómo me comunico con las autoridades? ¿Cómo entro a saber la base de proyectos para optar a fondos concursables?” (AU-PD-3); en la de las propias Líderes comunitarias "hablo por los demás, represento a la comunidad; yo vengo acá porque mi comunidad necesita esto, y apoyo para esto y esto otro" (LCDEC-D-3) y en las de Techo "muchos dirigentes también se quejan en el fondo que, claro, son líderes, representan a su comunidad pero las autoridades hacen oídos sordos de lo que ellos puedan decir y no hay espacios donde ellos sientan que participan” (DT-1).

En el espacio privado el conocimiento tanto para los profesores del diplomado como para las líderes comunitarias posibilita empoderarse en la intimidad del hogar, ante su pareja: "nos enseñaron de liderazgo (...). Aparte en mi caso, mi marido es machista, entonces saber que las mujeres eran 
tan importantes desde antes, porque siempre las mujeres están detrás, siempre está como bien escondida, es como el segundo plano, siempre detrás del marido, aquí [en el diplomado] no” (LCDEC-D-1).

Asimismo, se evidencia que las Líderes comunitarias por una parte transitan como personas y líderes entre estos dos espacios, público-privado (íntimo) y al mismo tiempo posibilitan la interjección de los mismos en su formación.

El papel transformador comunitario del conocimiento se alcanza mediante la formación de líderes y se materializa en dos tipos de liderazgos, uno dinamizador de la realidad comunitaria y otro que hemos denominado puentes en el bien entendido que canalizarían las demandas de sus comunidades hacia las autoridades y llevan información de las autoridades hacia los campamentos. El dinamizador se evidencia en Techo: “ella [la Directora de Educación] es la embajadora de educación en los campamentos y de una u otra forma se convierten en un ejemplo o debería para el resto de la comunidad” (DT-3) y en las líderes comunitarias "porque quiero ayudar a la gente, quiero que la gente tenga lo suyo, que tenga (...) que luchen por tener su casa, si tienen problemas, que los resuelvan, que le llegue la solución (...) eso me lleva a seguir en el cargo” (LCDEC-D-2). El liderazgo puente se evidencia en algunas voces de la universidad "entiendo a estos líderes comunitarios como aquellas personas que tienen un rol en estas comunidades de diversa naturaleza en pos de ayudar a salir adelante, en pos de implementar un tipo de política vecinal, comunal, digamos, como los espacios de poder intermedio y de contacto directo con la realidad" (AU-PD-3); de Techo "es ser voz de algunas familias que no pueden llegar a ciertas puertas” (DT-6) y de las líderes “Como líder he tenido que traspasar del sitio de donde estamos al municipio y tuve que enfrentarme con toda la gente de la comunidad (...) para que ellos me autoricen a firmar los papeles del municipio. Se logró eso, se consiguió en sitio de bienes raíces ya paso a la municipalidad” (LCC-1)

Otro papel social que se le atribuye al conocimiento es el sociopolítico. Este habilita para dialogar con el orden establecido en la sociedad, se observa socialmente neutro, implica riegos y contribuye a superar la pobreza.

La habilitación para el diálogo con el orden establecido se evidencia en algunas voces de la universidad "queremos que al final una persona (...) pueda fortalecer sus capacidades, ese es nuestro objetivo” (DU-1), en la líderes comunitarias "nos enseñaron a no ponernos coloradas, a no ponernos nerviosas, a no tener miedo a enfrentarse al público” ( LCDEC-D-2), "hemos hecho proyectos, ahora presentamos un proyecto para hacer una sede y yo ahora tengo voz y voto para hacer proyectos, puedo opinar, por ejemplo, yo fui al Techo a presentar el proyecto (LCDEC-D-1) y en Techo "para postular a fondos concursables, a miles de instancias principalmente gubernamentales que te permiten acceder a nuevos recursos” (DT-3).

Desde el papel social del conocimiento se advierte que para la uni- 
versidad el conocimiento sería socialmente neutro, esto es aséptico, evidenciándose en el documento de la Reforma universitaria ya referido (Zavala, 1966).

En las voces se advierten tres riesgos del papel sociopolítico del conocimiento: la mantención del statu quo, la instrumentalización ideológica y el levantamiento de líderes no naturales. La mantención del statu quo se advierte desde Techo “de esta forma el clasismo ampara el inmovilismo social, que es aún más injusto que la desigualdad social, pues el inmovilismo es quien alimenta la desigualdad social. Las personas quedan para siempre marcadas por el lugar de nacimiento, por características físicas o culturales. Se podrán hacer esfuerzos contundentes para que haya mayores oportunidades y permitir más movilidad social, pero si persiste el clasismo, se frenará todo tipo de iniciativas que busquen oportunidades más democráticas” (Berríos, 2006: 14). Tras este riesgo algunas voces de la universidad advierten que es posible caer en un paternalismo "siento que igual había un nivel de paternalismo en las personas llevar el pescado, así como este es el mensaje y tienen que llevarlo ustedes portadores del cambio” (AU-PD-3). Un segundo riesgo, advertido por la universidad, es instrumentalización del conocimiento con fines ideológicos "algunos [profesores] lo veían con un carácter más político, no sé si partidista pero si un rol más político, más que un rol facilitador respecto a la formación de niños y niñas de ese lugar, que era un poco la idea original” (AU-PD-3). Finalmente, el riesgo de que el conocimiento construido a través de la formación de líderes, haga surgir o levante líderes no naturales es señalado por los propios líderes comunitarios: "Creo que eso va en uno mismo, tratar de llegar a la gente con proyectos hechos que se van a hacer, que se van a concretar. Yo puedo tener cualquier diplomado de títulos, pero si no tengo vocación para llegar a la gente, de dirigirme a ellos para poderlos convencerlos, no va a servir de nada” (LCC-1).

Dentro del papel sociopolítico del conocimiento se establece que éste puede contribuir a la superación de la pobreza y hacerlo desde dos perspectivas: una instrumental y otras de construcción de comunidad. La de carácter instrumental es planteada por los tres actores. Desde la universidad se indica "mi idea [de la formación] es que la gente se siente digna y que logre desarrollar las herramientas para que salga adelante” (AU-PD-3); Techo refiere "queríamos fortalecer esta figura [Directoras de Educación] y fortalecerla también entregándoles los conocimientos suficientes a ella para que pudiesen mejorar su rol en el campamento (...) Entonces, la expectativa era que este diplomado le diese esas herramientas para cuando nosotros les propusiésemos un trabajo, ellas también pudiesen tener conocimientos para poder ejecutarlo (DT-2) y las líderes indican "para poder apoyar los TAC, en explicarles, en darles las herramientas, explicarles que para qué esto, por qué tienen que hacerlo. Yo me siento capacitada” (LCDEC-C-2). La segunda perspectiva, de construcción de la comunidad es referida por las líderes: "nosotros los cursos que tomamos son para ayudarnos a una comunidad completa, a nosotros mismos, pa' vivir mejor” (LCDEC-D-3) y por Techo "Hicimos un gran esfuerzo por transmitir que la erradicación de los campa- 
mentos no sólo consiste en el paso a la vivienda definitiva, sino que exige un proceso que rompa la verdadera lucha que es la exclusión social. Para ello, junto a pobladores y voluntarios, definimos una senda básica de recorrido -que llamamos Hoja de Ruta- que fija algunos mínimos en el proceso de desarrollo de las familias de campamentos. El gran sentido de esta ruta, a la que invitamos a voluntarios y pobladores, consiste en reducir al mínimo los espacios de exclusión en nuestra sociedad” (Domínguez, 2007: 12).

El cuarto papel sociopolítico del conocimiento es el intelectual y es reconocido sólo desde las dos instituciones, por una parte como transformador personal y por otro como transformador institucional. En la universidad se evidencia este papel intelectual como transformador personal, "nuestro proceso tiene que ver con fortalecer a las personas, fortalecer las oportunidades, las capacidades y que puedan tener una mejor vida" (DU-1) y en los documentos fundacionales de la PUCV (Castro, 1928), ya referidos. Así también la Universidad y Techo, reconocen que este papel intelectual es transformador de instituciones. En efecto ambas instituciones reconocen su propia transformación "[el diplomado] es un aporte a pensar juntos y a sentir juntos que queremos construir una mejor sociedad" (DT-1); "nosotros como universidad estamos en este camino y vuelvo a mencionar la palabra proceso porque es un proceso de aprendizaje, y de aprendizaje rico para ambas instituciones: esa es nuestra forma de colaborar y apoyar esta formación y este potenciamiento de capacidades y habilidades" (DU-1). Asimismo, se advierte la posibilidad de que ambas transformaciones se encuentren, en tanto no parecen excluyentes, sino complementarias.

\section{¿Qué aporte le atribuyen al conocimiento para la superación de las injusticias sociales?}

Tras el análisis de las fuentes es posible afirmar que existen tres aportes del conocimiento para la superación de la pobreza: individual, comunitario, y social/ institucional.

El aporte individual permitiría mejorar oportunidades laborales, democratizar oportunidades y que subsista la esperanza de movilidad social. Ciertamente, la universidad indica que aporta a la mejora de oportunidades laborales, entendiendo que este es el camino para llegar a una movilidad social: "Según consta en los documentos fundacionales, la Universidad se crea con el propósito explícito de preparar para el trabajo a personas de escasos medios económicos, en el contexto de una ciudad de marcado carácter comercial e industrial que carecía de establecimientos de enseñanza superior” (PUCV 2009: 13). Techo sustenta que democratiza oportunidades "justamente es a través de la educación y a lo largo del proceso de la educación formal en la que uno puede salir adelante, mejorando su situación y obtener mayor oportunidades" (DT-4); y a la vez, que permite que subsista la esperanza de la movilidad social: "La movilidad social se da por la educación, está estadísticamente demostrado solo por el ingreso de los salarios (...) yo creo que darle oportunidad a la gente para que pueda seguir aprendiendo y que ese aprendizaje lo pueda formalizar a través de progra- 
mas, cursos, talleres me parece como súper valioso” (AU-PD-3). Este aporte a la esperanza también lo manifiestan las líderes comunitarias: “que nos enseñen otras cosas, un oficio (LCDEC-D2); “estarse siempre capacitando para enseñarle a los demás” ( LCDEC-D1).

El aporte comunitario posibilitaría la formación de líderes, mejorar la calidad de vida y la habilitación para el diálogo con el poder establecido. En efecto, los tres actores señalan que es a través de la formación de líderes como se alcanzaría la superación de las injusticias sociales. La universidad, plantea que en coherencia con su sello valórico, esta formación debería integrarse transversalmente en el currículo de los estudiantes universitarios de pregrado "pienso que la universidad debería tener transversalmente esta formación de líderes comunitarios, porque la formación de liderazgo significa la formación en valores, y la formación en valores es sumamente compleja (AU-3) como de formación continua "la Universidad debiera generar (...) programas permanentes y progresivos (...) de apoyo en la formación de líderes comunitarios y eso implica programas permanente de largo aliento, que se comprometa con múltiples aspectos” (AU-PD-3). Techo valora la formación para la autoestima de las líderes comunitarias "ellas se sintieron muy validadas, (...) importantes, yo creo que a nivel de autoestima les ayudó mucho y la autoestima evidentemente ayuda a que ellas puedan desarrollar proyectos” (DT-1) y su interés por continuar perfeccionándose "que ellas tuvieran una buena experiencia que les sirviera para seguir con ganas de perfeccionarse” (DT-3). Para las líderes, su formación es importante para su desarrollo personal y el de sus comunidades "estarse siempre capacitando para enseñarles a los demás” (LCDEC-D-1); “uno tenía que tener liderazgo para organizarse como comunidad, ser líder para que la gente te escuche” (LCDEC-D-3). Al mismo tiempo, esta formación de líderes puede encaminarse hacia una formación de líderes transformadores o hacia aquella de líderes puentes, coincidentemente con respuesta (ya explicitada y sustentada) en la pregunta anterior, referida al papel del conocimiento.

El aporte referido a la mejora de la calidad de vida es recogido de algunas voces de Techo "La habilitación social del Techo tiene que ver con entregar herramientas, conocimientos a las familias para que puedan ellos mismos, a través de la autogestión de proyectos, mejorar su condición de vida” (DT-2) y de la universidad “fomentar sus competencias, su liderazgo, expresión oral de pararse, estoy pensando en cómo ellos tienen que defenderse desde sus vidas hasta sus negocios, sus ideas” (DU-1), “creo que es la labor que la universidad debería tener: es como la forma de compromiso para transformar un poco la sociedad, para que sea más justa, yo creo que eso es un deber no solo de la universidad sino que de la sociedad completa, para denunciar inequidades y pobreza” (AU-PD-3).

Respecto del aporte comunitario referido al diálogo con el poder establecido, cabe señalar que ya fue sustentado y explicitado en las respuestas evidenciadas en la pregunta sobre el papel social atribuido al conocimiento, emanadas desde los tres tipos de representantes. 
El aporte del conocimiento para la superación de la pobreza referido a lo social/ institucional, se observa desde tres dimensiones, la social, la institucional y la conjunción entre ambas. Desde la social, el conocimiento permite vincularse con el mundo comunitario: "Somos la única Universidad Pontificia que tiene una misión y una vocación social con sus alumnos, con sus profesores, y no podemos desconocer lo que sucede en nuestros medios, en nuestros medios más cercanos y en la sociedad en su conjunto" (DU-1). Sin embargo, hay un reconocimiento por parte de las instituciones de que no siempre se dispone de los conocimientos necesarios para cumplir con este papel. Si bien Techo destaca que posee un conocimiento de la realidad de los campamentos "la ventaja que tenemos nosotros de conocer el territorio constantemente" (DT-1), también se reconoce que "nadie sabe qué hay que enseñar" (DT-3). Para algunos actores de la universidad es necesario especializarse: "requiere que la universidad, y hablo de universidad como figura única, la gente que se dedica a esto o que le guste, debiera formarse, aprovechando la universidad, investigarlo, reflexionarse, introducirse, meterse en la realidad un poco más cercana de este tipo, y no solamente observarla desde la lejanía (AU-PD-3).

El no disponer de lo necesario para cumplir el papel de vinculación con el medio puede deberse a varias razones. Una de ellas, al desconocimiento de la realidad de los campamentos. Techo reconoce que "es muy difícil poder generalizar en la formación de todos los líderes, no todas las comunidades necesitan a los mismos líderes, ni los mismos tipos de liderazgos” (DT-4) y algunos profesores de la universidad se preguntan: “¿Qué se supone que es un líder comunitario? ¿Qué se supone que haga un líder comunitario? ¿Qué lo define como tal? Cosa de poder ver si finalmente estamos contribuyendo a eso o no" (AU-PD-2). Otra razón, a la diversidad del lenguaje, las líderes comunitarias manifiestan "[el profesor] usa unas palabras muy difíciles entonces uno no le entendía” (LCDEC-D1). Una tercera razón deriva, de los planteamientos de Techo que observa que en ocasiones el conocimiento no es aplicable al contexto "capacitación (...) para postular a fondos concursables, que es un tema que no tiene nada que ver con la educación misma, pero yo creo que desde el ámbito académico se le puede dar una estructura más concreta” (DT-3). Otra razón derivada de los planteamientos de los profesores es la disciplina y el desafío de la interdisciplinariedad "creo mucho en el trabajo interdisciplinario, por lo tanto el estar ahora trabajando con periodistas, con asistentes sociales y nosotras como educadoras, tenía hartas expectativas de lo que yo podría aprender" (AU-PD-2).

De las voces de las líderes comunitarias emerge que sólo ellas son quienes portan el conocimiento social necesario para vincularse con el medio comunitario y ello les da legitimidad ante ambas instituciones. Por otra parte, ellas validan el papel del conocimiento en la superación de la pobreza, legitimándolo: "Si ellos [los profesores] se interesan en una gente de una toma, que ellos se suponen que son a otro nivel, ná que ver con nosotros y que se interesen en trabajar con los campamentos, es bueno, porque así nos sube de nivel” ( LCDEC-D-2). 
La dimensión institucional del aporte del conocimiento a la superación de la pobreza se expresa de dos formas: en la metareflexión para transformarse y en la formación de líderes.

Sobre la metareflexión Techo indica que: “Quizás el rol que puedan tomar las universidades, como acercar todo este mundo que existe de la universidad hacia lugares que no tienen contacto directo, pero que sí debieran ser parte de” (VT-3). Para la universidad, esta metareflexión debiera ser interdisciplinaria "me parece que el trabajo que logramos ir construyendo también fue un aprendizaje para nosotros en el sentido de poner en discusión ciertas visiones también del trabajo particularmente con las dirigentes, de cuál es el lugar de la universidad en esta relación (...) los equipos tiene que ir construyendo en la interdisciplina” (AU-PD-1).

El aporte que se le atribuye al conocimiento para la superación de las injusticias sociales, desde un nivel institucional estaría dado en la formación de líderes, como se ha mencionado en los párrafos anteriores. Finalmente, la responsabilidad social universitaria emerge como una alternativa que permitiría a la universidad articular la tensión social-institucional "creo que se hace necesario que las universidades estén metidas con la realidad de las ciudades y que también, aparte de que gane la universidad, también es una responsabilidad que tiene la Universidad, o sea, se habla mucho de responsabilidad social universitaria pero efectivamente estos son el tipo de actividades que tienen que hacer las universidades” (DT-2).

\section{Discusión y conclusiones}

Los resultados muestran que las voces de los participantes de cada institución no son únicas sino diversas entre sí. Sin embargo, en todas ellas hay coincidencia respecto de que el conocimiento puede cumplir un rol de transformación social. Esta coincidencia aparece como el principal facilitador de la formación de líderes comunitarios y una oportunidad para su desarrollo.

Asimismo, llama la atención que en las voces de todos los participantes no esté presente la pregunta por el tipo de conocimiento más apropiado para generar transformación social. Esta ausencia parece fundamentarse en una concepción de conocimiento de tipo universal y que por lo tanto no se cuestiona. Dado los resultados, este es el conocimiento que tendría más legitimidad social para los participantes de las tres entidades. Cuestión que coincide con los planteamientos de Lander (1993) y Mignolo (2001).

Por otra parte, los resultados evidencian que la pregunta por la pertinencia del conocimiento, sigue estando vigente y que para pensar el tipo de conocimiento más apropiado para la formación universitaria de líderes comunitarios, aparece útil una metareflexión colectiva, la ampliación de la concepción de conocimiento y la explicitación de lógicas de poder. 
En efecto, se requiere una metareflexión conjunta, que permita vincular a los participantes involucrados, re-conociendo (Fraser, 2000) y revalorando el imaginario que cada cual ha construido. Asimismo, en la medida que la universidad amplíe su concepción de conocimiento y considere los imaginarios (Kozlarek, 2007) como rutas validas de comprensión, podría realizar un real aporte a la justicia social. Para que esta valoración y reconocimiento se dé, resulta apropiado explicitar las lógicas de poder que operan en estos procesos de formación. La universidad, dada su capacidad de certificar, y por esa vía legitimar, es vista por los participantes de las tres entidades como la que tiene más poder en esta relación, cuestión que coincide con la metáfora de la distinción (Bourdieu, 1989).

Los resultados también evidencian que en la medida que la universidad se vincule con el medio social y dialogue con los “otros”, podrá cumplir con su vocación de servicio y se re-humanizará. Efectivamente, la relación universidad-comunidad constituye por una parte, un eje de conexión entre la teoría y la práctica y al mismo tiempo, le permite el re-encuentro con su mito fundador, presente en los documentos fundacionales. Más aún cuando se evidencia que en la universidad se aprecia cierta deshumanización.

Por los resultados pareciera que la universidad no parece dispuesta ni recibe exigencias para moverse de la concepción de conocimiento universal tan legitimado socialmente. Por lo tanto, queda abierta la pregunta por la posibilidad de re-humanizarla.

Finalmente es posible afirmar que la formación universitaria de líderes comunitarios se presenta como una oportunidad de aprendizaje de carácter multidisciplinar e interinstitucional, y desafía a la Universidad a abrir su comprensión hacia otros imaginarios. La metareflexión destruiría el encapsulamiento y posibilitaría la construcción conjunta, ya lo proponía Tonon (2012).

En definitiva sobre la base de los resultados el equipo asume que el conocimiento es el resultado de un proceso de construcción dialógico (Panikkar, 2006), y no de una pura introspección individual, lo que implica dar reconocimiento al otro como un legítimo sujeto-otro de conocimiento. Para esto, se requiere que la universidad disponga de un tipo de conocimiento que está dispuesto a dialogar con otras epistemes, y no uno que clausure el diálogo por comprenderse como de mejor calidad.

Así, se asume el llamado a indisciplinar las ciencias (Wallerstein, 2007) como una acción necesaria para el desarrollo de la universidad, ya que al ubicar las disciplinas como la consecuencia directa de una determinada concepción de mundo, el trabajo inter o transdisciplinario se levanta como una vía apropiada para alcanzar un conocimiento de mejor calidad.

Es decir, la formación universitaria de líderes comunitarios, puede ser vista como una oportunidad de aprendizaje por parte de la propia universidad, si gracias a este proceso se avanza hacia la complementariedad 
entre la dialoguicidad y la interdisciplinariedad. Así, los dirigentes comunitarios obtendrían no sólo mayor conocimiento, sino también el re-conocimiento que ofrece la certificación universitaria. Así también, la universidad, recibe otro aporte para superar la visión eficientista de la educación superior, ya que desde el vínculo universidad-comunidad puede emerger una comprensión situada del conocimiento: desde el lugar (Escobar, 1993) comprendido desde la antropología, según indica Ysern (2007) para el bienestar. Esto permite sostener que el conocimiento pertinente, o situado, es aquel que se pone al servicio de una sociedad determinada y su noción de futuro. 


\section{Notas}

${ }^{1}$ Proyecto de Investigación DI-SV 037.83/2012, ejecutado entre abril y diciembre de 2012 y financiado por la Vicerrectoría de Investigación y Estudios Avanzados de la Pontificia Universidad Católica de Valparaíso (PUCV) en su Línea Sello Valórico. Los autores constituyen el equipo de investigadores y agradecen la colaboración técnica de la profesora Carolina Badillo y las ayudantes Pamela Altamirano y Valeria Viancos.

${ }^{2}$ TECHO es el nuevo nombre de la fundación chilena Un techo para Chile (UTPCH). Sobre Techo ver en: http://www.techo.org/en

${ }^{3}$ Imaginarios, de acuerdo a la propuesta de Appadurai (citado por Kozlarek, 2007:13) corresponden a campos organizados de prácticas sociales.

${ }^{4}$ Así, por ejemplo, el pastor Henry Cortés (de la Corporación Nuevo Pacto Iglesia y Ministerio), justificó el interés de la iglesia evangélica en Chile por adquirir la Universidad del Mar, señalando que al no tener universidad propia, los pastores evangélicos "no tienen posibilidad de obtener un reconocimiento, de una licenciatura en Teología" (La Tercera, 26 de diciembre de 2012:10).

${ }^{5}$ Esto, en el sentido de Foucault (2000). 


\section{Bibliografía}

Berríos, F. (2006), Todo comenzó en Curanilahue, Techo para Chile, Santiago.

Bourdieu, P. (1989), La distinción. Criterio y bases sociales del gusto, Taurus, Madrid.

Castro, R. (1928), Discurso Inaugural. Universidad Católica de Valparaíso.

Chakrabarty, D. (1992), "Provincializing Europ: Postcoloniality and the critique of history”, en Cultural Studies, Vol. 6, Issue 3, pp. 337-357.

Dilthey, W. (1978), El mundo histórico, Fondo de Cultura Económica, México.

Domínguez, P. (2007), Memoria, Techo para Chile, Santiago.

Donoso, S. y Schiefelbein, E. (2007), “Análisis de los modelos explicativos de retención de estudiantes en la universidad: una visión desde la desigualdad social”. Revista Estudios Pedagógicos, Valdivia: 33(1), 7-17.

Escobar, A. (1993), "El lugar de la naturaleza y la naturaleza del lugar: ¿globalización o postdesarrollo?”, en La colonialidad del saber: eurocentrismo y ciencias sociales. Perspectivas latinoamericanas, editado por E. Lander, pp. 113-143. CLACSO, Buenos Aires.

Esquivel, J. E. (2007) “Chile: Campo experimental para la reforma universitaria”, Perfiles Educativos, 29 (16), 41-59.

Feyerabend, P. K. (1989), Contra el Método, Ariel, Barcelona.

Foucault, M. (2000), Vigilar y Castigar, Siglo XXI, Madrid.

Fraser, N. (2000), “¿De la redistribución al reconocimiento? Dilemas de la justicia en la era “postsocialista””, en New Left Review, 0, enero 2000, pp. 126-155.

Gadamer, H. G. (2003), Verdad y Método, Volumen 1, Sígueme, Salamanca.

Goetz, J.P. y Le compte, M.D (1984), Etnografía y Diseño Cualitativo en Investigación Educativa, Morata, España.

González, L. E. y Espinoza, O. (2011) “La educación superior en Chile”, Pensamiento Universitario (22). Hernández, R. (2003) Metodología de la Investigación Social, Mc Graw Hill, México.

Hodder, I. (2000) “The interpretation of documents and material culture”, In Denzin, \& Lincoln (Eds.), Handbook of qualitative research (pp. 393402), Thousand Oaks, CA, Sage. 
Kozlarek, O. (coordinador), De la Teoría Crítica a una crítica plural de la modernidad, Biblos, Buenos Aires.

Lakatos, I. (2001), Historia de la ciencia y sus reconstrucciones racionales, Tecnos, Madrid, Reimpresión.

Lander, E., La colonialidad del saber: eurocentrismo y ciencias sociales. Perspectivas latinoamericanas, Buenos Aires, FLACSO.

Lorenz, (2010), Discurso Inaugural Año Académico de la Escuela de Pedagogía, Pontificia Universidad Católica de Valparaíso.

Manzano-Arrondo, V. (2012), La universidad comprometida, Bilbao, Hegoa.

Mato, D. (2010), “Las iniciativas de los movimientos indígenas en educación superior: un aporte para la profundización de la democracia”, en Nueva Sociedad $N^{\circ}$ 227, mayo-junio 2010.

Mato, D., coordinador (2009), Instituciones Interculturales de Educación Superior en América Latina, IESALC, Caracas.

Mayan, M. (2001), Una introducción a los métodos cualitativos: módulo de entrenamiento para estudiantes y profesionales. International Institute of Cualitative Methology, México.

Mella, O. (1998), Naturaleza y orientaciones teórico-metodológicas de la investigación cualitativa. Recuperado de http://ldei.ugr.es/doctorado/private/ cursos_pdf/investigacion\%20cualitativa.pdf

Mignolo, W. (2001), Capitalismo y Geopolítica del Conocimiento, Ediciones del Signo, Buenos Aires.

Morin, E. (2001), Introducción al pensamiento complejo, Gedisa, Barcelona.

Morin, E.; Roger, E. \& Motta, R. (2003), Educar en la era planetaria, Gedisa, España.

Osses, S.; Sánchez, I. \& Ibáñez, F.M. (2006) “Investigación Cualitativa en Educación. Hacia la generación de teoría a través del proceso analítico” en Revista Estudios Pedagógicos XXXII, Nº1, 119-133.

Panikkar, R. (2006), Paz e interculturalidad. Una reflexión filosófica, Herder, Barcelona.

Rodríguez-Ponce, E. (2011), “¿Por qué es necesario una reforma de la educación superior chilena?” en Idesia 29 (2):3-10. 
Sisto, V. \& Fardella, C. (2008), “Narrándose en la Flexibilidad. Un Análisis Narrativo Discursivo de la Identidad en Tiempos de Flexibilidad Laboral” en Revista de Psicología Vol. XVII, N² 2, pp 59-180.

Techo (2011-2012), Techo para Educación y Trabajo, Manual, Santiago.

Techo (2011), La voz de los niños, niñas y adolescentes en campamentos. Análisis de las condiciones de vida desde la mirada de la Infancia, Fundación Un Techo para Chile, Santiago.

Tonon, G. (2012) "Las relaciones universidad-comunidad: un espacio de reconfiguración de lo público”, en Polis vol.11, n.32, pp. 511-520.

Wallerstein, I. (2007), Impensar las ciencias sociales: límites de los paradigmas decimonónicos, Siglo XXI, México.

Walsh, C. (2007), “¿Son posibles unas ciencias sociales/culturales otras? Reflexiones en torno a las epistemologías decoloniales”, en Nomadas. (26): 102-113.

Ysern, J. L. (2007), “Comunicación y desarrollo en la experiencia de radio Estrella del Mar”, en VVAA, Sociedad y cultura, reflexiones transdisciplinarias, Universidad Austral de Chile.

Zabala, A. (1966), Discurso Inauguración Año Académico, Universidad Católica de Valparaíso.

Ídem (1966), Discurso Acto Clausura Temporada Académica, Universidad Católica de Valparaíso.

Recibido: 09.01.2013

Aceptado: 15.11.2013 